\title{
Capacity analysis of suburban rail networks
}

\author{
F. Rotoli ${ }^{1}$, G. Malavasi ${ }^{2}$, S. Ricci ${ }^{2}$, E. C. Navajas ${ }^{1}$ \& J. N. Ibáñez ${ }^{1}$ \\ ${ }^{I}$ European Commission, Joint Research Centre (JRC), \\ Institute for Prospective Technological Studies (IPTS), Spain \\ ${ }^{2}$ University of Rome "La Sapienza", Department of Civil, \\ Building and Environmental Engineering, Italy
}

\begin{abstract}
As is well known, capacity evaluation and the identification of bottlenecks on rail networks are complex issues depending upon several technical elements. This is even more perceptible in metropolitan areas where different services (freight, long distance, metro/regional, etc.) are operated using the same limited infrastructures; as a consequence, these facilities may represent bottlenecks of the rail system since they are often highly utilized and congested. This paper tries to explore the issue of capacity evaluation of complex rail networks, proposing synthetic indicators and analyses for feasibility studies or strategic planning. The presented methodology suggests taking into account the main differences in infrastructure characteristics (e.g. single or double lines, signalling systems, terminus or passing stations, etc.) and rail services (e.g. diverse rolling stock, various frequencies, average distances and number of stops, etc.) in order to propose a general approach applicable for capacity analysis of a network as a whole, hence evaluating the utilization rate and the congestion on both lines and stations. To better explore and validate the methodology, an application to a line of the Naples' suburban network is presented. The results confirm the applicability and effectiveness of the proposed approach; the outcomes indicate the capacity utilization rate of the considered facilities, pointing out likely bottlenecks and possible actions to improve the system efficiency.
\end{abstract}

Keywords: capacity, railway network, suburban railway system, bottlenecks.

\section{Introduction}

In the last decade the European Union has been trying to promote a modal shift towards rail being seen as one of the keys for the sustainable development of a 
more competitive and resource-efficient transport system, mainly due to its low external and environmental costs (European Commission [1]).

The significant efforts devoted in improving the competitiveness of the rail mode at European scale are highlighted by the adoption of the four railway packages and their related directives; after having opened to competition the markets for rail freight services and for international passenger transport (long distance), currently the European Commission is focusing also on national markets for domestic passenger transport services (i.e. regional, small-scale) which remain largely closed and are still considered the bastions of national monopolies.

In this context, rail network capacity and bottlenecks are becoming a cause of concern for policy makers, particularly in certain corridors as increasing traffic eventually leads to congestion and degraded performance of the railway system.

Clearly, the capacity of rail infrastructure is a complex issue depending upon several factors; beside others, the benefits of creating a transnational method for its assessment are highlighted in the International Union of Railways (UIC) Code 406 [2]. Indeed, in the last years the scientific literature has devoted great efforts in trying to address this issue; many contributions provide an accurate distinction (synthetic, analytical, simulation models) and description of different methodologies (see [3-7]). Several approaches address the assessment of line capacity (as described in [8]); Landex et al. [9], for example, focus on the application of the UIC code 406 while [10] and [11] describe the Capacity Utilization Index (CUI) procedure applied in UK. Other authors analyse the issue at station level: Malavasi et al. [12] provide a review of capacity methods for complex railway nodes and a detailed description of some synthetic approaches; Lindner [13] tries to extend the applicability of the UIC Code 406 even to the terminals. Also the UIC, in recent studies, presents a net distinction between line [14] and node [15] capacity, reporting a comparative analysis of different synthetic or analytical methodologies for their evaluation.

Regarding the rail system as a whole, it is not straightforward to give a unique measure of capacity because of complexity and diversification of components (lines, stations or their subparts) but it is possible to estimate a global capacity value by referring to the lower local values. Indeed, several papers focus also on the issue of capacity at network level; for example [16] suggest an analytical approach while [17] a queuing model for capacity assessment of a railway system.

The approach presented in the next paragraphs utilizes synthetic methods (for their flexibility and easy applicability) and proposes a joint analysis of nodes and lines in order to allow identifying bottlenecks among of all the features of the network.

\section{Line's analysis}

The capacity of a line's segment between consecutive stations is estimated through the analytical method proposed in its first edition by the International Union of Railway (UIC) in the leaflet 405R (see [8] or [18]). To summarise briefly the main characteristics of this approach, it is based on the following formula: 


$$
P=\frac{T}{t_{f m}+t_{r}+t_{z u}}
$$

- $\quad P$ is the capacity (daily, hourly etc.);

- $\quad T$ is the reference time;

- $t_{f m}$ is the average minimum headway;

- $t_{r}$ is an expansion margin;

- $t_{z u}$ is an extra time based on the number $a$ of the intermediate block sections on the line's segment and it is calculated by means of the formula $t_{z u}=0.25^{*} a$; this parameter takes into account that the increase of capacity on the determinant section, following its division into more block sections, is less than proportional to the reduction of the travel time.

The expansion margin was introduced as a result of experiences of many European rail organizations (included UIC) to take into account the utilization of the system. This margin is expressed as a rate of the average minimum headways between convoys; for short periods of time (peak hour), common values of this rate vary between 0.3 and 0.4 , while for longer periods (full day) usually values between 0.6 and 0.8 are adopted.

The average minimum headway for each line is calculated by using a weighted average of the minimum headway between two consecutive trains of the same category:

$$
\underline{t}_{f m}=\frac{\alpha_{L} * t_{f m L}+\alpha_{R} * t_{f m R}+\alpha_{M} * t_{f m M}}{\alpha_{L}+\alpha_{R}+\alpha_{M}}
$$

In particular, the procedure considers three different typologies of train: longdistance passenger trains (L), local/regional passenger trains $(\mathrm{R})$ and freight trains (M, this last category encloses also out-of-service and empty run); of course the factors $\alpha_{L}, \alpha_{R}$ and $\alpha_{M}$ in the previous formula represent the percentages of the categories on the total of trains.

In case of an automatic block signalling system with three aspects the minimum headways (as distance) between consecutive convoys and for each segment of double-track lines is constituted by a first block section (to guarantee the braking distance of the train and so safety conditions) plus a second block section (to guarantee not disrupted circulation, i.e. a running train should always find the approaching signal 'clear' to avoid unnecessary acceleration/deceleration phases and so a disturbed circulation) plus a distance for the sight of the signal and the clearing of the section and finally a distance equal to the train length for the release of block system.

In practice, the minimum headway for each category will be calculated as:

$$
t_{f m_{L, R, M}}=\frac{l_{b 1}+l_{b 2}+L}{V_{L, R, M}}+t_{s}
$$

where:

- $\quad l_{b 1}$ and $l_{b 2}$ represent the length of the block sections;

- $L$ the length of the convoy (assumed for all the trains equal to 500 meters);

- $V_{L, R, M}$ is the speed relative to the considered category;

- $t_{s}$ is the sum of the sighting and clearing times (assumed equal to 30 seconds). 


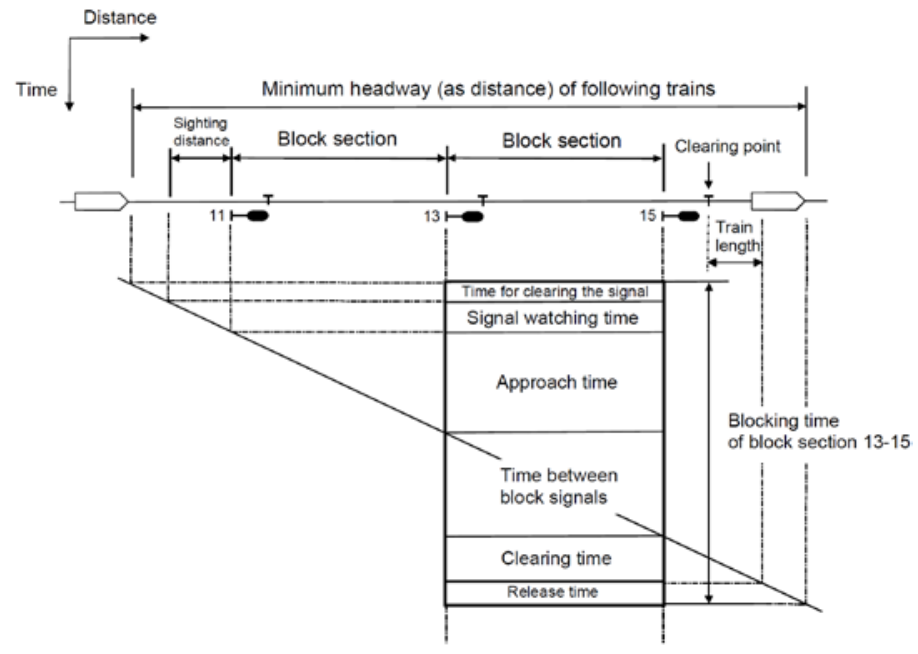

Figure 1: Scheme for the calculation of the blocking time for double-track lines by [3].

For the single-track lines, instead, each segment between two stations can be occupied only by a train, independently from its running direction. In this case the minimum headway (as time) for each category can be calculated as:

$$
t_{f m_{L, R, M}}==t_{V_{L, R, M}}+t_{a}+t_{d}+t_{p}
$$

where

- $t_{V L, R, M}$ represents the travel time with constant speed:

$$
t_{V_{L, R, M}}=\frac{l_{b}}{V_{L, R, M}}
$$

- $\quad t_{a}$ and $t_{d}$ represent the acceleration and deceleration times:

$$
t_{a_{L, R, M}}=\frac{V_{L, R, M}}{a}, t_{d_{L, R, M}}=\frac{V_{L, R, M}}{d}
$$

- $t_{p}$ represent an extra time for the preparation of the itinerary.

\section{Station's analysis}

The stations can be considered as features with a variable topology due to the presence of switches, with the exception of the halt station, here defined as a terminal with a fixed configuration (i.e. only the main tracks/platforms, see Figure 2(a)) and allowing for passengers' services.

A net distinction shall be made between terminus and passing stations (see Figures 2(b) and 2(c)) based on configuration and type of offered services; the former ones usually present higher dwelling times, being characterized by a change in the running direction of trains and terminus services for some routes (i.e. entering and exiting switches areas are overlapped, with consequent higher utilisation of the same infrastructure and more incompatibilities between incoming 
and outgoing paths), while the latter present a configuration with two distinct zones for the entrance and the exit of trains.
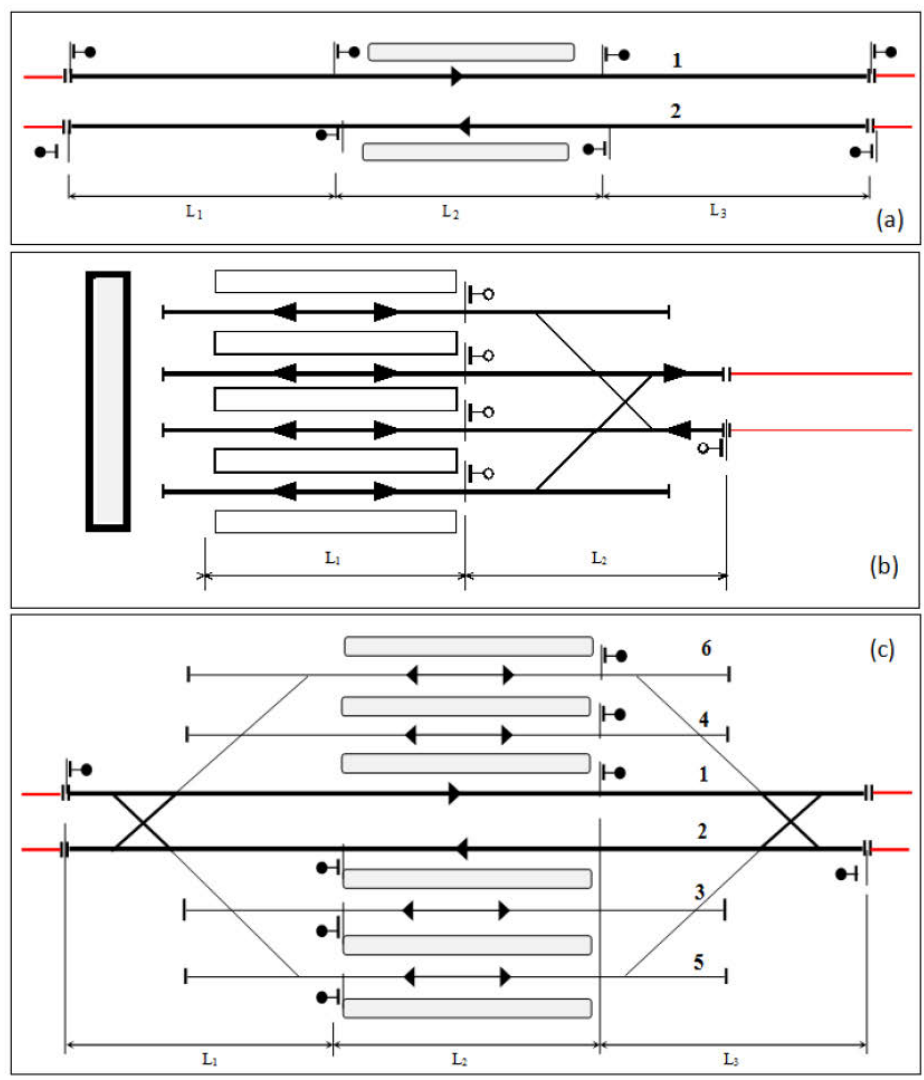

Figure 2: Standardized schemes of the stations: (a) halt station, (b) terminus station, (c) passing station.

In particular, since it is not always easy to get access to the detailed schematic plan for each station of a network, we assumed in our application a 'standard' configuration of them (see Figure 2), varying only the number of lateral platforms.

The halt station (see Figure 2(a)) is treated as part of a line, so embedded in a block section; this means that we can calculate the capacity with equation (1) where in the expression of the minimum headway times, for the trains stopping in the terminal, we consider also the accelerating/braking times and the dwelling times. In our case study (see next paragraph) we have assumed the same length of 1000 meters for $L_{1}, L_{2}$ and $L_{3}$ in Figure 2(a).

For the capacity evaluation of passing stations, the procedure utilizes a synthetic approach, i.e. Potthoff method (see [12] or [19]). This method assumes that trains could arrive at any instant of an assigned time period (T) with the same probability; it does not require an assigned timetable because the methodology is 
based on a global quantitative analysis of the traffic in the period T. Its great advantage is the simplicity of application.

Practically, based on a fixed topological configuration of the station (see Figure 2(c), where we have assumed the same length of 750 meters for $L_{1}, L_{2}$ and $L_{3}$ ) and varying only the number of lateral (siding) platforms, we have analysed the incompatibility among of the possible routes and calculated the average number of compatible routes:

$$
\underline{n}=\frac{N^{2}}{\sum\left(n_{i} * n_{j}\right)}
$$

where:

- $\quad N$ : total number of movements $\left(N=\Sigma n_{i}=\Sigma n_{j}\right)$;

- $\quad n_{i}$ : number of movements concerning the route $\mathrm{i}$;

- $\quad n_{j}$ : number of movements concerning the route $\mathrm{j}$;

- the summation in the denominator is extended to all the couples of incompatibles routes.

The percentages of services stopping at specific platforms can be obtained by the station timetables (as done in the application described in the next paragraph) which indicate the planned platform for each train. Beside the average number of compatible routes (based mainly on the topological configuration of the station and on the percentage of trains per each route), the method requires also the determination of the average interdiction time between incompatible routes, calculated again as weighted average on the categories of trains:

$$
\underline{t}_{i}=\frac{\alpha_{L}{ }^{*} \underline{t}_{i L}+\alpha_{R}{ }^{*} \underline{t}_{i R}+\alpha_{M} * t_{i M}}{\alpha_{L}+\alpha_{R}+\alpha_{M}}
$$

For each category, the average interdiction time is obtained by a weighted average of the interdiction times for each couple of incompatible routes:

$$
\underline{t}_{i L, R, M}=\frac{\sum\left(n_{i} * n_{j} * t_{i j L, M, R}\right)}{\sum\left(n_{i} * n_{j}\right)}
$$

The interdiction times between routes are calculated based on the assumed topological configurations of the stations and for services stopping in the terminal, they are given by:

$$
t_{i j_{L, R, M}}==t_{V_{L, R, M}}+t_{a}+t_{d}+t_{s}+t_{p}
$$

where $t_{V L, R, M}$ represents the travel time with constant speed, $t_{a}$ and $t_{d}$ represent the acceleration and deceleration times, $t_{s}$ represents the dwelling time and $t_{p}$ represents an extra time for the preparation of the itinerary.

In reality, depending on the type of incompatibility between the two routes and on the assumed topology, the interdiction times have been assumed as sum of either all the factors in equation (10) or only part of them; e.g. for passing services we have considered only the travel time at constant speed and the extra time for the formation of the route.

Finally, the coefficient of utilization of the station (namely $U$ ) is determined in function of the total occupation time (indicated with B in the following formula) and the total operating time $(T)$ by means of the following equation: 


$$
U=\frac{B}{T}=\frac{N}{\underline{n}} * \frac{t_{i}}{T}
$$

\section{Case study: Naples - Battipaglia line}

To better explore the applicability and the potentiality of the proposed methodology, we have also carried out a more specific and detailed analysis of the Naples-Battipaglia line [20], from the Naples' suburban rail network (see Figure 3 ); this line is part of the main rail corridor connecting Naples with the south of Italy, in particular with Calabria and Sicily. It includes several parallel sections (see Figure 3) with different characteristics and travelled by various types of passenger trains (High Speed, Intercity and Regional):

- the conventional line from Napoli Centrale to Salerno passing by Torre Annunziata is mainly used by regional trains and it is further divided in two (double-track and electrified) lines between Nocera Inferiore and Salerno; in detail the section via Cava dei Tirreni is a complementary line offering mostly local services;

- the high-capacity and high-speed line from Napoli Centrale to Salerno passes by P.C. Vesuvio and reconnects with the traditional line at Bivio Santa Lucia; High Speed trains run on it. Since the High Capacity line is still interested by a limited number of trains and it is not exactly part of the Neapolitan suburban network, we focused only on the more congested and critical traditional line.
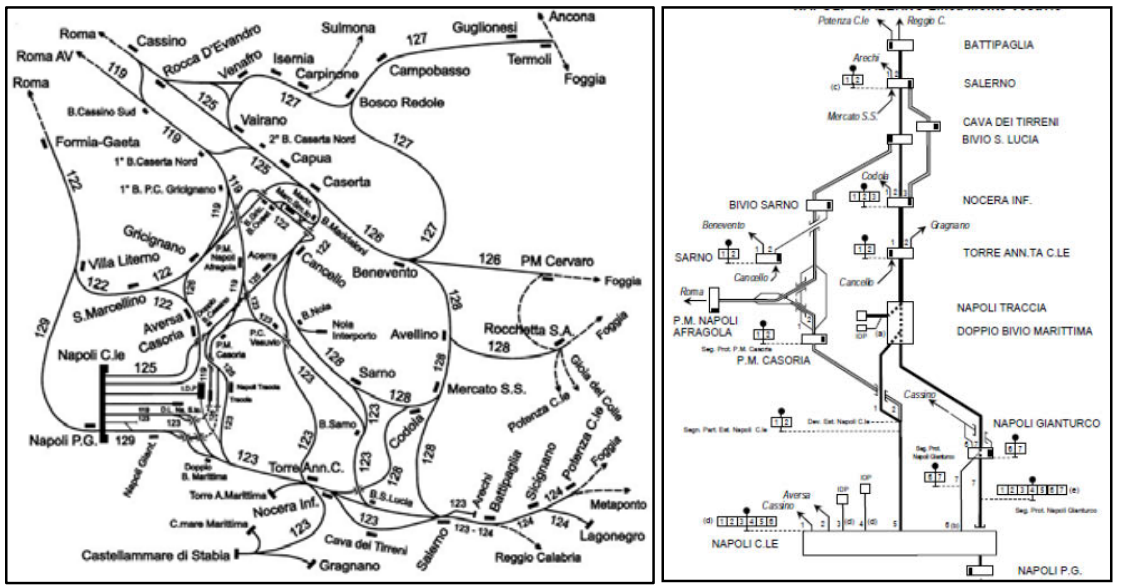

Figure 3: Schematic layout of the Naples' rail network (left) and of the NaplesBattipaglia line (right) [20].

Detailed data related both to the infrastructure and to the timetable [21] for all the Italian lines are available, and free downloadable from the RFI (Rete Ferroviaria Italiana) and Trenitalia websites (Figure 4); based on this data it is 
possible to obtain the block sections' lengths, the maximum allowed speed for each category of convoy (the operational plan of the line, namely 'Fascicolo Linee' [20], reports three categories - A, B and C - of speeds relative to freight, regional and long distance trains), the number of trains per segment and the number of train stopping in each station. Moreover, from the stations' timetables, always available on the same website, it is possible to know the number of used platforms in each terminal and the percentage of trains assigned to them.

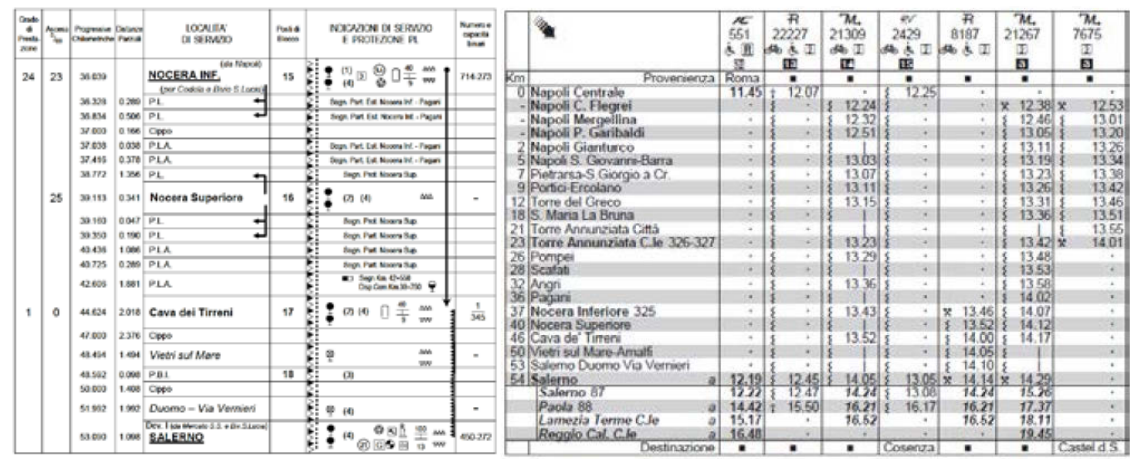

Figure 4: Extracts of the schematic infrastructure plan [20] (left) by RFI and timetable [21] (right) for the Naples-Salerno line by Trenitalia.

With all these figures, it is possible to proceed with the already described approach for both stations and lines; the only information missing is related to the number of freight trains. We have assumed an additional percentage of $10 \%$ of the total number of trains per link or station to take in account the freight, the out-ofservice and/or the empty circulations.

Figure 5 reports for each direction of the analysed line the utilization rate per section; in particular, the lengths of the block sections in this case are well known and the ranges in the graphs correspond to a buffer time (i.e. $t_{r}$ ) equal to $60 \%$ (lower limit) or $80 \%$ (upper limit) of the average minimum headway (i.e. $t_{f m}$ ). The results show that the most critical sections (25 from Naples towards Battipaglia while 10 and 11 in the opposite direction) correspond to the segment between Bivio S. Lucia and Salerno, characterized by block sections' length of around $5 \mathrm{~km}$ in both the directions and travelled by High Speeds, InterCitys, and part of the Regional trains (in addition to the $10 \%$ of the total assumed for freight, out-ofservice and empty services).

Moreover, Figure 6 reports the utilization rates for the stations along the line, with daily operating time of 20 hours and dwelling times of 1,2 or 3 minutes; of course we have neglected Naples Central, since it is a terminus station with a quite complex configuration and operating timetable, and thus it deserves a separate and specific analysis. 


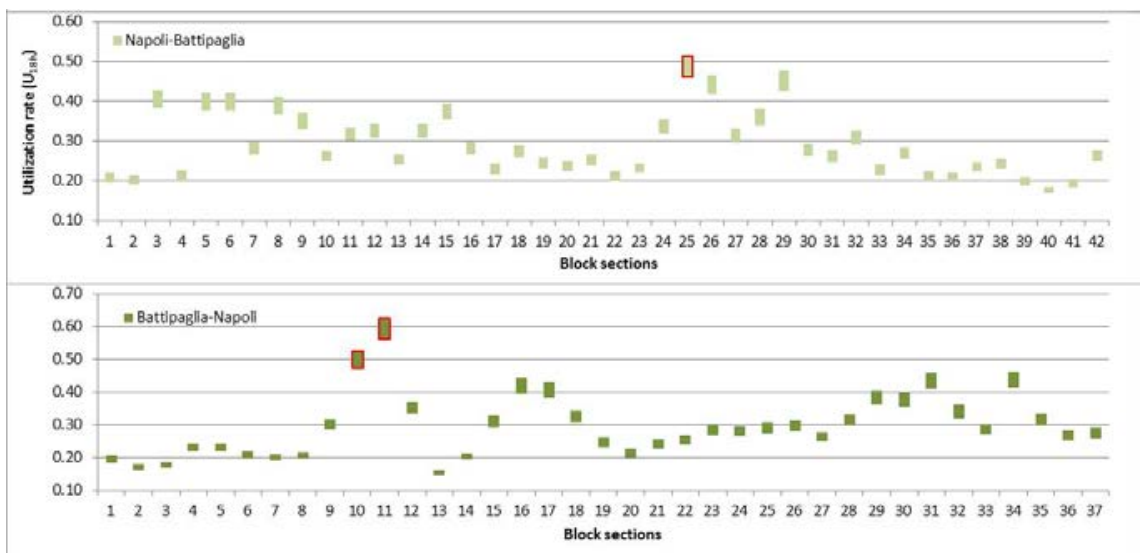

Figure 5: Variability of utilization rates per direction and per section for the Naples-Battipaglia line; lower and upper limits corresponding respectively to a buffer time $\left(t_{r}\right)$ of 0.6 or 0.8 of the average minimum headway $\left(t_{f m}\right)$.

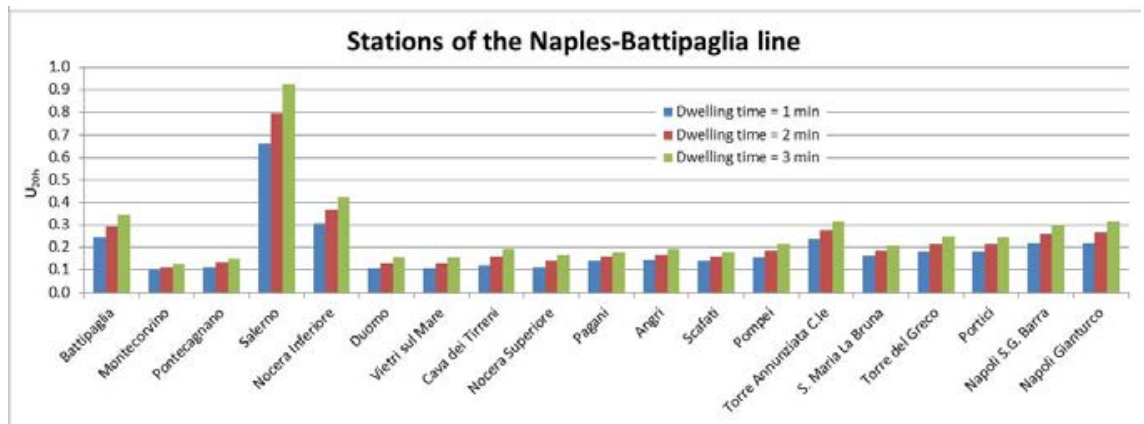

Figure 6: Utilization rates of the stations of the Naples-Battipaglia line, with daily operating time of 20 hours and dwelling times of 1,2 or 3 minutes.

Looking at the results summarized in Figure 6, the station more utilized on the Naples-Battipaglia corridor is represented by Salerno which seems to be quite congested and the only significantly critical along the whole line; in reality despite the high number of trains interesting the station, its configuration is quite complex, as showed in Figure 7 from OpenRailwayMap (www.openrailwaymap.org/).

It is characterized by terminus tracks/services and different line segments (we have analysed the ones from Nocera Inferiore via Bivio Santa Lucia, from Nocera inferiore via Cava dei Tirreni and towards Battipaglia; see also Figure 3). Thus, as even indicated by the results of our procedure, this station (similarly to the Naples one) should be subjected to a specific and more detailed examination. 


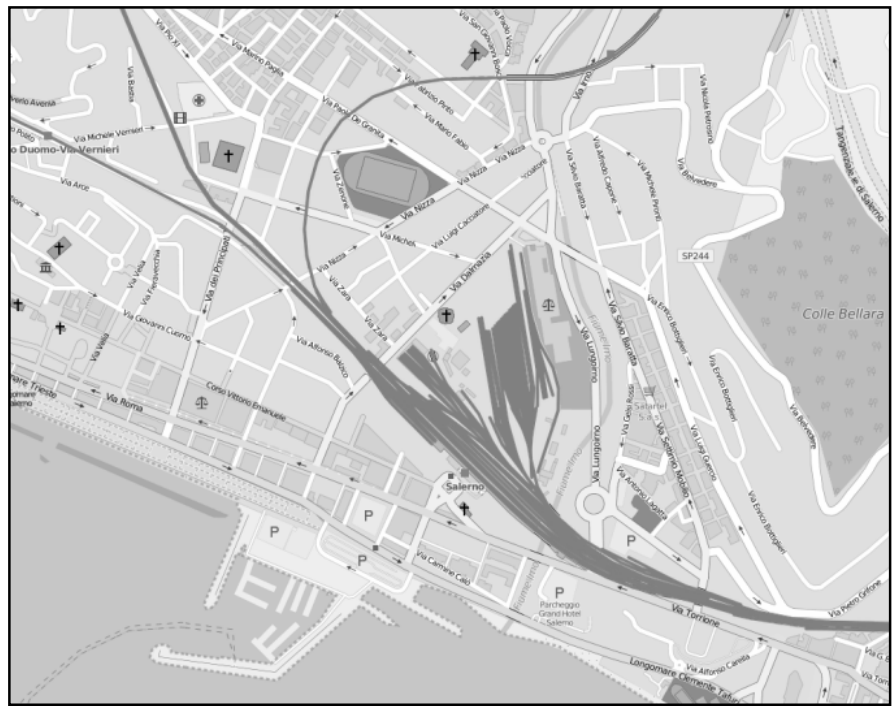

Figure 7: Salerno station from OpenRailwayMap.

\section{Conclusion}

This contribution proposes a synthetic methodology for the utilisation assessment of suburban rail networks. In particular, the approach proposes the identification and the schematization of the elementary features of the network (stations and line's segments) in order to provide a joint analysis of nodes and links.

To better evaluate the applicability and the soundness of the proposed approach, a case study based on actual data for the Naples-Battipaglia line is presented. The application shows that the methodology allows indicative evaluations on the use of the system and comparative analysis between different features, providing a first identification of 'weak' links or nodes for which, then, specific and detailed analyses should be carried out, taking in account more in deep their actual configuration, the technical characteristics and the real composition of the traffic (i.e. other elements influencing the rail capacity, such as: the adopted operating systems, the cyclic clocking of the services, the station traffic/routes control and safety system, the elastic release of routes, the overlap of block sections, etc.).

\section{Disclaimer}

The views expressed are purely those of the authors and may not in any circumstances be regarded as stating an official position of the European Commission. 


\section{References}

[1] European Commission (2011). White Paper. Roadmap to a Single European Transport Area - Towards a competitive and resource efficient transport system.

[2] International Union of Railways (UIC), Leaflet 406R - "Capacity", June 2004.

[3] Hansen, I. \& Pachl, J. (2014). Railway Timetabling and Operations. Analysis - Modelling - Optimisation - Simulation - Performance Evaluation, 2nd edition. Eurailpress.

[4] Transportation Research Board (2013). Transit Cooperative Research Program Report 165, Transit Capacity and Quality of Service Manual, 3rd Edition. Chapter 8 - Rail Transit Capacity.

[5] Abril, M., Barber, F., Ingolotti, L., Salido, M. A., Tormos, P. \& Lova, A. (2008). An Assessment of Railway Capacity, Transportation Research Part E, Elsevier, pp. 774-806.

[6] Kontaxi, E. \& Ricci, S. (2009). Techniques and methodologies for carrying capacity evaluation: comparative analysis and integration perspectives. Ingegneria Ferroviaria, pp. 1051-1080, December 2009.

[7] Kontaxi, E. \& Ricci, S. (2012). Railway Capacity Handbook: A Systematic Approach to Methodologies. Transport Research Arena, Procedia - Social and Behavioral Sciences, Volume 48, pp. 2689-2696.

[8] Rotoli, F., Ricci, S., Navajas Cawood, E. \& Malavasi, G. (2015). Capacity versus Punctuality Assessment Procedures and Accessibility Measures for Rail Networks. Ingegneria Ferroviaria, Ed. CIFI. December 2015, pp. 1011-1040.

[9] Landex, A., Schittenhelm, B., Kaas, A. H. \& Schneider-Tilli, J. (2008). Capacity measurement with the UIC 406 capacity method Computers in Railways XI, WIT Transactions on The Built Environment, Volume 103.

[10] Gibson, S., Cooper, G. \& Ball, B. (2002). Developments in Transport Policy. The Evolution of Capacity Charges on the UK Rail Network. Journal of Transport Economics and Policy, Volume 36, Part 2, pp. 341354, May 2002.

[11] Faber Maunsell-Aecom, (2007). Capacity Tariff Charge PR2008. Network Rail, October 2007.

[12] Malavasi, G., Molková, T., Ricci, S. \& Rotoli, F. (2014). A synthetic approach to the evaluation of the carrying capacity of complex railway nodes. Journal of Rail Transport Planning \& Management, Volume 4, Issue 1-2, pp. 28-42, August-October 2014.

[13] Lindner, T. (2011). Applicability of the analytical UIC Code 406 compression method for evaluating line and station capacity, Journal of Rail Transport Planning \& Management, Volume 1, pp. 49-57, November 2011.

[14] International Union of Railways - UIC. (2008). Influence of ETCS on line capacity - Generic study.

[15] International Union of Railways - UIC. (2010). Influence of the European Train Control System (ETCS) on the capacity of nodes. 
[16] Mussone, L. \& Calvo, R. W. (2013). An analytical approach to calculate the capacity of a railway system. European Journal of Operational Research, Volume 228, Issue 1, pp. 11-23.

[17] Huisman, T., Boucherie, R. J. \& van Dijk, N. M. (2002). A solvable queueing network model for railway networks and its validation and applications for the Netherlands, European Journal of Operational Research, Volume 142, pp. 30-51.

[18] International Union of Railways - UIC. (1996). Code 405 OR - Links between Railway Infrastructure Capacity and the Quality of Operations.

[19] Potthoff, G. (1963-1972). Verkerhrsstnomungslehre 1. Transpress VEB Verlag für Verkehrswesen, Berlin.

[20] Rete Ferroviaria Italiana - RFI. (2016). Fascicolo Linea 123 (Naples Department). Edition 2003, Revision 022/2015. https://normativa esercizio.rfi.it/Default.aspx.

[21] Trenitalia. (2015). Orario Regionale Campania-Molise (pdf version, validity period: 13 December 2015-11 June 2016). www.trenitalia.com/ tcom/Informazioni/Orari-regionali-in-versione-pdf. 